\title{
The Usefulness of New-Generation Capsule Endoscopy in Patients with Portal Hypertensive Enteropathy
}

\author{
Seung-Joo Nam, Ji Hyun Kim and Sung Chul Park \\ Division of Gastroenterology and Hepatology, Department of Internal Medicine, Kangwon National University School of Medicine, \\ Chuncheon, Korea
}

See "Mucosal Changes in the Small Intestines in Portal Hypertension: First Study Using the Pillcam SB3 Capsule Endoscopy System" by Mahesh Kumar Goenka, Bhavik Bharat Shah, Vijay Kumar Rai, et al., on page 563-569.

Portal hypertension (PH) is a clinical syndrome of pathologically elevated portal systemic pressure, which is a hepatic venous pressure gradient of $>5 \mathrm{~mm} \mathrm{Hg}{ }^{1,2}$ It is caused by increased hepatic vascular resistance and increased portal inflow due to splanchnic vasodilatation. Liver cirrhosis (LC) is one of the most common causes of $\mathrm{PH}$, and $\mathrm{PH}$ may lead to ascites, portosystemic encephalopathy, and mucosal abnormalities of the gastrointestinal (GI) tract, including esophageal and gastric varices, portal hypertensive gastropathy (PHG), portal hypertensive colopathy (PHC), and portal hypertensive enteropathy (PHE). ${ }^{3}$ PHE refers to mucosal abnormalities of the small bowel and, more specifically, edematous and hyperemic lesions reminiscent of inflammation, but the exact definition of PHE still needs to be established. ${ }^{4,5}$ The pathological mechanism of the development of PHE has not yet been clearly identified. However, the ischemia-reperfusion phenotype, including vasodilatory response, has been considered the main cause, while the leukocytic phenotype, including infiltration by inflammatory cells and bacteria, and the angiogenic

Received: October 3, 2018 Revised: October 24, 2018

Accepted: October 31, 2018

Correspondence: Sung Chul Park

Department of Internal Medicine, Kangwon National University Hospital, Kangwon National University School of Medicine, 156 Baengnyeong-ro, Chuncheon 24289, Korea

Tel: +82-33-258-2405, Fax: +82-33-258-2146, E-mail: schlp@hanmail.net ORCID: https://orcid.org/0000-0003-3215-6838

(c) This is an Open Access article distributed under the terms of the Creative Commons Attribution Non-Commercial License (http://creativecommons.org/ licenses/by-nc/3.0) which permits unrestricted non-commercial use, distribution, and reproduction in any medium, provided the original work is properly cited. phenotype, including epithelial remodeling and submucosal angiogenesis have also been suggested. ${ }^{6} \mathrm{PHE}$ is mostly asymptomatic but may occasionally cause GI bleeding or anemia.

The classification of PHE by De Palma et al. ${ }^{7}$ has been widely used, where grade 1 refers to mucosal inflammatory-like abnormalities, including edema, erythema, granularity, and friability, while grade 2 refers to vascular lesions such as cherry-red spots, telangiectasia, angiodysplasia-like lesions, and varices. In addition, Abdelaal et al. ${ }^{8}$ classified PHE as inflammatory-like lesions, red spots, angioectasias, and small bowel varices. Kodama et al. ${ }^{9}$ classified PHE into villous abnormalities (edema, atrophy, and reddening) and vascular lesions (angiodysplasia-like lesions, dilated/proliferated vessels, and varices).

The reported prevalence of PHE, mostly caused by LC, ranges from $18.2 \%$ to $96.8 \% .^{5,7,8,10-15}$ Many studies that used capsule endoscopy (CE) as a diagnostic test for obscure GI bleeding reported that the prevalence of PHE was $>60 \%$. A multicenter study conducted in Korea using data from the Capsule Endoscopy Nationwide Database Registry, which included $45 \mathrm{LC}$ and $\mathrm{PH}$ patients, reported a PHE prevalence of $40 \% .{ }^{13}$ In general, vascular lesions such as red spots or angiodysplasia-like lesions are more common than non-vascular/inflammatory lesions. ${ }^{2,13}$ The prevalence rates of small bowel varices and active bleeding have been reported to range from $8.1 \%$ to $38.9 \%$ and from $5.5 \%$ to $17.8 \%$, respectively. ${ }^{2,7,11,13,16,17}$

The risk factors associated with PHE include esophageal varices, $\mathrm{PHG}$, PHC, Child-Turcotte-Pugh class B or C, porto- 
systemic shunts, previous endoscopic sclerotherapy or ligation for varices, a history of acute GI bleeding, high liver fibrosis index, and high computed tomography scores based on the $\mathrm{PH}$ findings such as varices, $\mathrm{PHG}, \mathrm{PHC}$, splenomegaly, and ascites. ${ }^{2,5,7,8,11-13,18}$ However, the reported PHE prevalence and related factors vary according to the studies, and most reports were of single-center studies, which were limited by small numbers of patients.

Currently, no standard therapeutic guideline exists for symptomatic PHE. Balloon-assisted enteroscopy, radiological interventions such as transjugular intrahepatic portosystemic shunt placement or percutaneous coil embolization, and surgery may be used. ${ }^{1}$ The relevant endoscopic procedures include argon plasma coagulation, hemoclipping, polypectomy, and variceal injection sclerotherapy. Although no extensive studies have been conducted on PHE, non-selective beta-blockers or somatostatin may be used as medications for $\mathrm{PH}$, and a case in which thalidomide administration $(100 \mathrm{mg} /$ day) led to the successful normalization of hemoglobin levels was reported..$^{19}$

CEs developed for observing the inner lumen of the small bowel include PillCam SB (Given Imaging, Yoqneam, Israel), MiroCam (IntroMedic, Seoul, Korea), EndoCapsule (Olympus, Tokyo, Japan), OMOM (Jinshan Science, Chongqing, China), and CapsoCam (CapsoVision, Saratoga, CA, USA). ${ }^{20}$ PillCam $\mathrm{SB}$ was developed into the second-generation $\mathrm{SB} 2$, and recently, the third-generation SB3 was introduced. The most important improvement in SB3 is its ability to capture images at an adaptive frame rate of two to six frames per second rather than the fixed two frames per second of SB2. ${ }^{21}$ Such an improvement maximizes the visible small bowel mucosa, which is especially advantageous when the capsule passes through the proximal part of the small bowel, which is known for its quick passage time. The resolution has also been improved in SB3, enabling the acquisition of brighter, clearer, and detailed images of the small bowel. The battery life of the capsule has also been increased to up to 12 hours.

In this issue of Clinical Endoscopy, Goenka et al. ${ }^{22}$ evaluated the small bowels of patients with $\mathrm{PH}$ of various etiologies who had symptoms of unexplained anemia or occult GI bleeding, using the PillCam SB3 CE system, focusing on PHE. Abnormal findings were categorized into vascular (red spots, telangiectasia, or varices) and non-vascular/inflammatory lesions (villous edema, erythema, or polyps). A CE score of 1 point was given to each finding if they were solitary and 2 points were given if they were numerous. The correlations between CE scores and clinical, laboratory, and endoscopic parameters were also determined. Among the 43 patients included in the study, 41 (95.3\%) showed abnormal features, including varices (67.4\%), red spots (60.5\%), villous edema (46.5\%), erythe- ma (44.2\%), telangiectasia (16.3\%), and polyps (16.3\%). The proximal small bowel was the most common site of involvement (34 patients). In this study, the prevalence of PHE was investigated for the first time using only the PillCam SB3 CE system and was found to be as high as $95.3 \%$. In particular, the detection rate of small bowel varices was $67.4 \%$, which was higher than that in other studies. This may be attributed to the high detection rate of pathological lesions by PillCam SB3 CE, as suggested by the authors. Although this study proved the effectiveness of SB3 CE in PHE detection, the lack of comparison with a control group, in which SB2 was used, makes it difficult to determine any significant improvement. Some studies that compared SB2 and SB3 reported that SB3 shows a significantly reduced reading time and a significantly improved detection rate, while other studies have reported no significant differences between SB2 and SB3. ${ }^{21,23-27}$ In addition, some of the polyps classified as PHE in the study by Goenka et al. ${ }^{22}$ may be tumorous lesions such as adenomas, and not inflammatory changes. Thus, further studies are needed to analyze whether PHE detection is indeed increased with the use of SB3.

The CE score for PHE ranged from 0 to 8 (mean \pm standard deviation, 4.09 \pm 1.8 ), and patients with virus-related liver disease and a history of GI bleeding showed higher PHE scores. However, a lack of evidence makes it difficult to determine if the viral etiology of LC aggravated PH more than other non-viral causes, and it is presumptuous to assume so from this study alone, as only 12 patients had virus-related LC. Although this study included a univariate analysis of factors related to PHE scores, a multivariate analysis seemed not to be performed, possibly because of the insufficient number of study subjects. Thus, the possibility that variables related to GI bleeding such as age or sex served as confounding factors should be considered.

Among the 43 patients in the study, five (11.6\%) showed evidence of ongoing or recent bleeding related to PHE. Of these five patients, three received endoscopic treatment, while one received coil embolization. The management of symptomatic PHE lesions should be approached with consideration of the patient's clinical state, available treatment methods, and expertise of the medical center.

This study showed that the prevalence of PHE was high in patients with $\mathrm{PH}$, suggesting that SB3 CE could effectively detect lesions related to PHE, such as varices. Therefore, although further studies are needed, new-generation CE may be useful in evaluating and managing anemia or obscure GI bleeding in patients with PH. Large-scale prospective studies are needed to determine the prevalence of PHE in patients with $\mathrm{PH}$, identify clinical factors related to $\mathrm{PHE}$, and provide appropriate management for clinical settings. 
Conflicts of Interest

The authors have no financial conflicts of interest.

\section{REFERENCES}

1. Jeon SR, Kim JO. Capsule endoscopy for portal hypertensive enteropathy. Gastroenterol Res Pract 2016;2016:8501394.

2. Mekaroonkamol P, Cohen R, Chawla S. Portal hypertensive enteropathy. World J Hepatol 2015;7:127-138.

3. Rondonotti E, Villa F, Signorelli C, de Franchis R. Portal hypertensive enteropathy. Gastrointest Endosc Clin N Am 2006;16:277-286.

4. Kalafateli M, Triantos CK, Nikolopoulou V, Burroughs A. Non-variceal gastrointestinal bleeding in patients with liver cirrhosis: a review. Dig Dis Sci 2012;57:2743-2754.

5. Kovács M, Pák P, Pák G, Fehér J, Rácz I. Small bowel alterations in portal hypertension: a capsule endoscopic study. Hepatogastroenterology 2009;56:1069-1073

6. Aller MA, Arias JL, Cruz A, Arias J. Inflammation: a way to understanding the evolution of portal hypertension. Theor Biol Med Model 2007;4:44.

7. De Palma GD, Rega M, Masone S, et al. Mucosal abnormalities of the small bowel in patients with cirrhosis and portal hypertension: a capsule endoscopy study. Gastrointest Endosc 2005;62:529-534.

8. Abdelaal UM, Morita E, Nouda S, et al. Evaluation of portal hypertensive enteropathy by scoring with capsule endoscopy: is transient elastography of clinical impact? J Clin Biochem Nutr 2010;47:37-44.

9. Kodama M, Uto H, Numata $M$, et al. Endoscopic characterization of the small bowel in patients with portal hypertension evaluated by double balloon endoscopy. J Gastroenterol 2008;43:589-596.

10. Chandrasekar TS, Janakan GB, Chandrasekar VT, Kalamegam RY, Suriyanarayanan S, Sanjeevaraya PM. Spectrum of small-bowel mucosal abnormalities identified by capsule endoscopy in patients with portal hypertension of varied etiology. Indian J Gastroenterol 2017;36:32-37.

11. Figueiredo P, Almeida N, Lérias C, et al. Effect of portal hypertension in the small bowel: an endoscopic approach. Dig Dis Sci 2008;53:21442150.

12. Goulas S, Triantafyllidou K, Karagiannis S, et al. Capsule endoscopy in the investigation of patients with portal hypertension and anemia. Can J Gastroenterol 2008;22:469-474.

13. Jeon SR, Kim JO, Kim JB, et al. Portal hypertensive enteropathy diagnosed by capsule endoscopy in cirrhotic patients: a nationwide multicenter study. Dig Dis Sci 2014;59:1036-1041.
14. Tsai CJ, Sanaka MR, Menon KV, Vargo JJ. Balloon-assisted enteroscopy in portal hypertensive enteropathy. Hepatogastroenterology 2014;61:1635-1641.

15. Aoyama T, Oka S, Aikata H, et al. Major predictors of portal hypertensive enteropathy in patients with liver cirrhosis. J Gastroenterol Hepatol 2015;30:124-130.

16. Akyuz F, Pinarbasi B, Ermis F, et al. Is portal hypertensive enteropathy an important additional cause of blood loss in portal hypertensive patients? Scand J Gastroenterol 2010;45:1497-1502.

17. Canlas KR, Dobozi BM, Lin S, et al. Using capsule endoscopy to identify GI tract lesions in cirrhotic patients with portal hypertension and chronic anemia. J Clin Gastroenterol 2008;42:844-848.

18. Aoyama T, Oka S, Aikata $\mathrm{H}$, et al. Is small-bowel capsule endoscopy effective for diagnosis of esophagogastric lesions related to portal hypertension? J Gastroenterol Hepatol 2014;29:511-516.

19. Jimenez-Saenz M, Romero-Vazquez J, Caunedo-Alvarez A, Maldonado-Perez B, Gutierrez JM. Beneficial effects and reversion of vascular lesions by thalidomide in a patient with bleeding portal hypertensive enteropathy. Dig Liver Dis 2010;42:232-233.

20. Kwack WG, Lim YJ. Current status and research into overcoming limitations of capsule endoscopy. Clin Endosc 2016;49:8-15.

21. Monteiro S, de Castro FD, Carvalho PB, Moreira MJ, Rosa B, Cotter J. PillCam ${ }^{\mathbb{B}}$ SB3 capsule: does the increased frame rate eliminate the risk of missing lesions? World J Gastroenterol 2016;22:3066-3068.

22. Goenka MK, Shah BB, Rai VK, Jajodia S, Goenka U. Mucosal changes in the small intestines in portal hypertension: first study using the Pillcam SB3 capsule endoscopy system. Clin Endosc 2018;51:563-569.

23. Kim SH, Choi HS, Chun HJ, et al. Diagnostic benefit of simultaneous capsule endoscopy using two different systems. Gastroenterol Res Pract 2018;2018:9798546.

24. Kunihara S, Oka S, Tanaka S, et al. Third-generation capsule endoscopy outperforms second-generation based on the detectability of esophageal varices. Gastroenterol Res Pract 2016;2016:9671327.

25. Omori T, Hara T, Sakasai S, et al. Does the PillCam SB3 capsule endoscopy system improve image reading efficiency irrespective of experience? A pilot study. Endosc Int Open 2018;6:E669-E675.

26. Rahman M, Akerman S, DeVito B, Miller L, Akerman M, Sultan K. Comparison of the diagnostic yield and outcomes between standard $8 \mathrm{~h}$ capsule endoscopy and the new $12 \mathrm{~h}$ capsule endoscopy for investigating small bowel pathology. World J Gastroenterol 2015;21:5542-5547.

27. Xavier S, Monteiro S, Magalhães J, Rosa B, Moreira MJ, Cotter J. Capsule endoscopy with PillCamSB2 versus PillCamSB3: has the improvement in technology resulted in a step forward? Rev Esp Enferm Dig 2018;110:155-159. 